\title{
The centrally-symmetric solutions of electronic excitations of semiconductors in the conditions of relativistic like degeneracy of dynamical properties
}

\author{
A.D.Suprun, L.V.Shmeleva \\ Faculty of Physics, Kyiv National T. Shevchenko University, \\ 64/13, Volodymyrska Str., Kyiv, Ukraine, 01601
}

Received November 25, 2013

\begin{abstract}
The excited states of materials with the structure of crystals were analyzed in the paper. Here the reaction of crystalline lattice on excitation was taken into account. Such consideration leads to nonlinear Schr $\backslash$ "\{o\}dinger equations. At a solution of these equations some possible variants of the nonlinearities which are different from the cubic are considered. The possibility of constructing of spherically-symmetric analytical solutions with finite norm analyzed. The analytical solutions in the form of centrally symmetric solitons were found. The obtained solutions show the expressed wave-corpuscle dualism. It is shown, that dynamic properties of excitation are identical to dynamics of not selftrapped quasiparticle. An important feature of these solutions is that their amplitudes (or squares of the amplitudes) have an asymptotic behavior of the type of $1 / \rho$, with $\rho \rightarrow \infty$.
\end{abstract}

В работе проанализированы возбужденные состояния материалов со структурой кристаллов. Здесь учитывалась реакция кристаллической решетки на возбуждение. Такое рассмотрение приводит к нелинейным уравнениям Шредингера. При решении этих уравнений рассмотрено несколько возможных вариантов нелинейностей, отличных от кубической. Проанализирована возможность построения сферически-симметричных аналитических решений с конечной нормой. Получены аналитические решения в виде центрально-симметричных солитонов. Полученные решения демонстрируют выраженную корпускулярно-волновую дуальность. Также показано, что динамические свойства возбуждения тождественны не автолокализованной квазичастице. Важной особенностью полученных решений является то, что их амплитуды (или квадраты амплитуд) имеют степенную асимптотику типа $1 / \rho$, при $\rho \rightarrow \infty$.

Центрально-симетричні солітони електронних збуджень напівпровідників б умовах релятивістсько-подібного виродження динамічних властивостей. А.Д.Суnрун, Л.В.ШМельова. У роботі проаналізовано збуджені стани матеріалів зі структурою кристалів. Тут враховувалася реакція кристалічної гратки на збудження. Такий розгляд приводить до нелінійних рівнянь Шредінгера. При розв'язанні цих рівнянь розглянуто кілька можливих варіантів нелінійностей, що відрізняються від кубічної. Проаналізовано можливість побудови сферично-симетричних аналітичних розв'язків 3 кінцевою нормою. Отримано аналітичні розв'язки у вигляді центрально-симетричних солітонів. Отримані розв'язки демонструють виражену корпускулярно-хвильову дуальність. Також показано, що динамічні властивості збудження тотожні вільній квазічастинці. Важливою особливістю отриманих розв'язків $\epsilon$ те, що їх амплітуди (або квадрати амплітуд) мають ступеневу асимптотику типу $1 / \rho$, якщо $\rho \rightarrow \infty$. 


\section{Introduction}

Soliton excitations in solids are a development of the concept of traditional electronic excitations (the injected electrons, excitons) or excitations of other physical nature (magnons [1], polarons [2], and others like that). In materials of crystalline type excitation of solitons is conditioned by the local reaction of crystalline grate on excitation (one of conditions ((the third) of ideality of crystal). The main feature that distinguishes the soliton excitation from traditional one - is the appearance of amplitude, which is distributed in space and time.

This paper deals with the analytical solutions in the form of central-symmetric solitons for the nonlinear Schrödinger equation (NSE) with different types of nonlinearities. Was analyzed their properties, in particular, dynamic. It is important for the interpretation of the transfer of energy [3,4], charge [5] and other physical characteristics [6,7].

General dynamical properties of solitons based on one of the most important characteristics - on the dispersive dependence of energy or frequency from the wave vector [8-11]. Unlike a previous studies [12] and in the development of some of the other of them $[13,14]$ is taken into account the effects on these properties of the nonlinearities.

\section{Solitonic excitations in solids. General comments}

This research is a natural extension of research [12]. The simplest Hamiltonian of the electronic excitations in solids with the local response of the crystalline lattice on excitation can be reduced to such general form $[13,15]$ :

$$
\begin{aligned}
& E_{f}(\{a\})=(1 / 2)\left\{\sum_{\mathbf{n} \mathbf{l}}^{/} w_{\mathbf{n}, \mathbf{n}+\mathbf{l}}+\sum_{\mathbf{n}} D_{\mathbf{n}}^{f} \cdot\left|a_{f \mathbf{n}}\right|^{2}+\right. \\
& \left.+\sum_{\mathbf{n}} G_{\mathbf{n}}^{f}\left(\left|a_{f \mathbf{n}}\right|^{2}\right)+\sum_{\mathbf{n} \mathbf{l}} / M_{\mathbf{n}, \mathbf{n}+\mathbf{l}}^{f} \cdot\left(a_{f \mathbf{n}}^{*} a_{f, \mathbf{n}+\mathbf{l}}+a_{f, \mathbf{n}+\mathbf{l}}^{*} a_{f \mathbf{n}}\right)\right\} .
\end{aligned}
$$

The dash on the double sum means that the lattice vector $\mathbf{n}$ takes an arbitrary values and the lattice vectors $\mathbf{l}$ - all values except $\mathbf{l}=\mathbf{0}$. Factor $a_{f \mathbf{n}}$ determines the spatial-temporal distribution of excitation within bounds of crystal space and within the lifetime of the excitation. It is to be determined. In order to determine this factor and eigenvalues $E_{f}(\mathbf{k})$ the procedure of the dynamic Hamilton minimization [4,13] is used for the functional (1). Matrix elements $w_{\mathbf{n}, \mathbf{n}+\mathbf{l}}, D_{\mathbf{n}}^{f}$ and $M_{\mathbf{n}, \mathbf{n}+\mathbf{l}}^{f}$ for simple (or simple in approximation) crystals have precise definitions [13].

The function $G_{\mathbf{n}}^{f}\left(\left|a_{f}\right|^{2}\right)$ appears in the functional (1) due to the waiver of the third condition of crystal ideality, which is related to neglect of reaction of crystalline lattice on excitation. Its important property is that in the performance of the first two conditions of ideality of the crystal (infinity of space and absence of heterogeneity), it loses an external index $\mathbf{n}$. This property will to be always fulfilled. Near zero it has the following asymptotic properties actual in future. Its expansion in the series begins from a summand $\left(\left|a_{f \mathbf{n}}\right|^{2}\right)^{2} \equiv\left|a_{f \mathbf{n}}\right|^{4}$, if it does not have a symmetry, or if it have an even symmetry. If it has odd symmetry, decomposition begins from an element $\left(\left|a_{f_{\mathbf{n}}}\right|^{2}\right)^{3} \equiv\left|a_{f_{\mathbf{n}}}\right|^{6}$. "New" equilibrium values of vectors of crystalline grate $\mathbf{b}_{\mathbf{n}}^{f}$ in the case of weak reaction of lattice on excitation and depending on parity are related to "old" their values b by correlations:

$$
\mathbf{b}_{\mathbf{n}}^{f}=\mathbf{b}-\boldsymbol{\gamma}_{f_{\mathbf{n}}} \cdot\left|a_{f \mathbf{n}}\right|^{2}+\cdots, \text { or } \mathbf{b}_{\mathbf{n}}^{f}=\mathbf{b}-\gamma_{f \mathbf{n}} \cdot\left(\left|a_{f_{\mathbf{n}}}\right|^{2}\right)^{2}+\cdots
$$

The $\gamma_{f \mathbf{n}}$ parameters do not depend on the vector $\mathbf{n}$, if two conditions of ideality, which remained, are satisfied.

Considering only the situation further, when the first two conditions of ideality are executing the functional (1) can be reduced to a simpler form: 


$$
E_{f}(\{a\})=E_{f}^{(0)}+(1 / 2) \sum_{\mathbf{n}}\left\{G_{f}\left(\left|a_{f \mathbf{n}}\right|^{2}\right)+\sum_{\mathbf{l}(\neq \mathbf{0})} M_{\mathbf{1}}^{f} \cdot\left(a_{f \mathbf{n}}^{*} a_{f, \mathbf{n}+\mathbf{1}}+a_{f, \mathbf{n}+\mathbf{1}}^{*} a_{f \mathbf{n}}\right)\right\} .
$$

Here, the notation: $E_{f}^{(0)} \equiv U_{0}+D_{\mathbf{0}}^{f} N_{f} / 2$ - is a fixed part of the energy (3). Factor $U_{0} \equiv \sum_{\mathbf{n} \mathbf{l}} / w_{\mathbf{n} \mathbf{l}} / 2$ is the potential energy of the crystal, and the factor $N_{f} \equiv \sum_{\mathbf{n}}\left|a_{f \mathbf{n}}\right|^{2}$ is the norm of $a_{f \mathbf{n}}$ with respect to $\mathbf{n}$.

\section{Solitonic excitations in solids. Cubic nonlinearity}

This case, presumably, is the most investigated $[1,3,4,12-16]$. Therefore, it is easy to illustrate and to use farther fundamental aspects of statements of problems and its solutions for other variants of nonlinearities. This case corresponds to the situation of even symmetry of the $G_{f}\left(\left|a_{f \mathbf{n}}\right|^{2}\right)$ function, or absence of its symmetry, and representation: $G_{f}\left(\left|a_{f \mathbf{n}}\right|^{2}\right)=-\left(G_{f} / 2\right)\left(\left|a_{f_{\mathbf{n}}}\right|^{2}\right)^{2} \equiv-\left(G_{f} / 2\right)\left|a_{f_{\mathbf{n}}}\right|^{4}$, takes place. The multiplier $1 / 2$ is entered for comfort of subsequent consideration. In typical cases $G_{f}>0$. The functional (3) in such representation acquires a kind:

$$
E_{f}(\{a\})=E_{f}^{(0)}+(1 / 2) \sum_{\mathbf{n}}\left\{\sum_{\mathbf{l}(\neq \mathbf{0})} M_{\mathbf{1}}^{f} \cdot\left(a_{f \mathbf{n}}^{*} a_{f, \mathbf{n}+\mathbf{1}}+a_{f, \mathbf{n}+\mathbf{l}}^{*} a_{f \mathbf{n}}\right)-\left(G_{f} / 2\right)\left|a_{f \mathbf{n}}\right|^{4}\right\} .
$$

Using this functional in procedure of dynamic Hamilton minimization $[4,13]$ :

$$
i \hbar\left(\partial a_{f \mathbf{n}} / \partial t\right)=\partial E_{f}(\{a\}) / \partial a_{f \mathbf{n}}^{*}
$$

it is possible to get equation:

$$
i \hbar\left(\partial a_{f \mathbf{n}} / \partial t\right)+G_{f}\left|a_{f \mathbf{n}}\right|^{2} a_{f \mathbf{n}}+(1 / 2) \sum_{\mathbf{1}(\neq \mathbf{0})}\left|M_{\mathbf{1}}^{f}\right|\left(a_{f, \mathbf{n}+\mathbf{1}}+a_{f, \mathbf{n}-\mathbf{l}}\right)=0 .
$$

Here it was also taken into account that in typical crystals: $M_{1}^{f}=-\left|M_{1}^{f}\right|[13]$.

Since equation (4) is complex, it is necessary first of all to represent the function $a_{f \mathbf{n}}$ in the general form:

$$
a_{f \mathbf{n}}(t)=\varphi_{f \mathbf{n}}(t) \cdot \exp \left[i \cdot \Gamma_{f \mathbf{n}}(t)\right]
$$

After substituting (5) into (4) and separating the real and imaginary parts, the system (3) is divided in two subsystems to determine the amplitude $\varphi_{f_{\mathbf{n}}}$ and phase $\Gamma_{f_{\mathbf{n}}}$.

$$
\begin{gathered}
\hbar\left(\frac{\partial \phi_{f \mathbf{n}}}{\partial t}\right)+\frac{1}{2} \sum_{\mathbf{l}(\neq \mathbf{0})}\left|M_{1}^{f}\right|\left\{\phi_{f, \mathbf{n}+\mathbf{1}} \sin \left(\Gamma_{f, \mathbf{n}+\mathbf{1}}-\Gamma_{f \mathbf{n}}\right)-\phi_{f, \mathbf{n}-\mathbf{1}} \sin \left(\Gamma_{f \mathbf{n}}-\Gamma_{f, \mathbf{n}-\mathbf{1}}\right)\right\}=0 ; \\
\hbar \phi_{f \mathbf{n}}\left(\frac{\partial \Gamma_{f \mathbf{n}}}{\partial t}\right)=G_{f} \phi_{f \mathbf{n}}^{3}+\frac{1}{2} \sum_{\mathbf{l}(\neq \mathbf{0})}\left|M_{\mathbf{l}}^{f}\right|\left\{\phi_{f, \mathbf{n}+\mathbf{l}} \cos \left(\Gamma_{f, \mathbf{n}+\mathbf{1}}-\Gamma_{f \mathbf{n}}\right)+\phi_{f, \mathbf{n}-\mathbf{1}} \cos \left(\Gamma_{f \mathbf{n}}-\Gamma_{f, \mathbf{n}-\mathbf{1}}\right)\right\} .
\end{gathered}
$$

We will continue to work in a generalized approximation of a plane wave in phase:

$$
\Gamma_{f \mathbf{n}}(t)=\mathbf{k}(t) \cdot \mathbf{n}-\gamma_{f}(t) .
$$

The difference of this generalized representation from that of [12] in the fact that the wave vector $k(t)$ and the energy factor $\gamma_{f}(t)$ (instead of the product $\omega_{f} t$ ) are arbitrary functions of time. Their explicit form is determined by the features of the dynamics of the examined excitations. 
In applications usually works in, so-called, continuum approximation [12], in which is limited to representation:

$$
\varphi_{f, \mathbf{n} \pm \mathbf{l}}=\varphi_{f}(\mathbf{n}) \pm\left(\mathbf{l} \cdot \nabla_{\mathbf{n}}\right) \varphi_{f}(\mathbf{n})+(1 / 2)\left(\mathbf{l} \cdot \nabla_{\mathbf{n}}\right)^{2} \varphi_{f}(\mathbf{n})
$$

From point of approximations considered in [12] it will be the fourth approximation.

Before discussing system (6), (7) farther, we will simplify it by means of previous three approximations which were discussed in detail in [12]. The first of it is approximation of flat wave in a phase (it is already used in representation (8)). The second of it - is the approximation of the nearest neighbors. And, finally, the third approximation - is the approximation of a cubic lattice.

For comfort of the further use of continuum approximation, was entered also the identical notations: $\mathbf{n} \equiv b x_{\alpha} \mathbf{e}_{\alpha}$. Variables $x_{\alpha}$ have the sense of dimensionless spatial coordinates and are the components of vector $\mathbf{r}$. Hence: $\mathbf{n} \equiv b \mathbf{r}$. Denotations of work [12] are used also for constants $C_{f}$ and $m_{f}$, the first of which make sense of the maximum speed of quasiparticle: $C_{f}=(b / \hbar)\left|M_{f}\right|$, and second make sense of scalar mass:

$$
m_{f}=\hbar^{2} /\left(b^{2}\left|M_{f}\right|\right)
$$

There was re-designated also: $\left|M_{b}^{f}\right| \equiv\left|M_{f}\right|$. In analogy to [12], equations (6), (7) can be reduced to dimensionless form. This can be done by an additional (with respect to the space variables) definition of the dimensionless time $\tau=\left(\left|M_{f}\right| / \hbar\right) t$ and the dimensionless wave momentum $\mathbf{p}=b \mathbf{k} \quad\left(p_{\alpha}=k_{\alpha} b\right)$. Also were used the notation of [12] for the dimensionless velocity $\beta_{\alpha}=\sin \left(p_{\alpha}\right)$ and dimensionless dynamic mass $\mu_{\alpha} \equiv 1 / \cos \left(p_{\alpha}\right)$. Then, finally, equations (6) (7) take the form:

$$
\begin{gathered}
\partial \varphi_{f} / \partial \tau+\beta_{\alpha} \cdot\left(\partial \varphi_{f} / \partial x_{\alpha}\right)=0 \\
\left(1 / 2 \mu_{\alpha}\right)\left(\partial^{2} \varphi_{f} / \partial x_{\alpha}^{2}\right)+g_{f} \varphi_{f}^{3}+\left(\sum_{\alpha} 1 / \mu_{\alpha}+\left(\dot{\gamma}_{f}-\dot{\mathbf{p}} \cdot \mathbf{r}\right)\right) \varphi_{f}=0 .
\end{gathered}
$$

In equation (12) used also a denotation:

$$
g_{f}=G_{f} /\left|M_{f}\right|
$$

Denotations $\dot{\gamma}_{f}$ and $\dot{\mathbf{p}}$ now mean derivatives on dimensionless time $\tau$.

The general solution of equation (11) for the function $\varphi_{f} \equiv \varphi_{f}(\tau, \mathbf{r}) \equiv \varphi_{f}\left(\tau,\left\{x_{\alpha}\right\}\right)$ is an arbitrary function of such structure:

$$
\varphi_{f}(\tau, \mathbf{r})=\varphi_{f}\left(\mathbf{r}-\mathbf{r}_{0}(\tau)\right) \equiv \varphi_{f}\left(\left\{x_{\alpha}-x_{0}^{(\alpha)}(\tau)\right\}\right) \equiv \varphi_{f}\left(\left\{\xi_{\alpha}\right\}\right) \equiv \varphi_{f}(\boldsymbol{\xi})
$$

A vector $\mathbf{r}_{0}(\tau)$ is determined here by dynamic equation:

$$
\dot{\mathbf{r}_{0}}=\boldsymbol{\beta}(\mathbf{p})
$$

or in components:

$$
x_{0}^{\dot{\alpha})}=\beta_{\alpha}\left(p_{\alpha}\right)=\sin \left(p_{\alpha}\right)
$$

and actually makes sense of equation of trajectory of point $\mathbf{r}_{0}$ in the crystalline system of co-ordinates, if the vector of wave momentum $\mathbf{p}$ is defined, as function of time $\tau$. Equation (12) then takes the form of the nonlinear Schrödinger equation:

$$
\left(1 / 2 \mu_{\alpha}\right)\left(\partial^{2} \varphi_{f} / \partial \xi_{\alpha}^{2}\right)+g_{f} \varphi_{f}^{3}-\dot{\mathbf{p}} \cdot \boldsymbol{\xi}+\left(\sum_{\alpha} 1 / \mu_{\alpha}+\left(\dot{\gamma}_{f}-\dot{\mathbf{p}} \cdot \mathbf{r}_{0}\right)\right) \varphi_{f}=0
$$


already formulated in own coordinate system associated with the point $\mathbf{r}_{0}(\tau)$. In the examined approximation of a plane wave in phase, in spite of its generality, there are no grounds for the use of dynamic conditions on the momentum $\mathbf{p}$, different from those of $\dot{\mathbf{p}}=0$. Other conditions in the absence of external influences (fields) may occur, if to give up the approximation of plane waves in phase. In simplest case in sense of continuum approximation of kind of (9) not only in relation to amplitude $\varphi_{f}$ but also in relation to a phase $\Gamma_{f}[16,17]$, in equation (12) and, as a result, in equation (17) there will be the elements of type of the integrated non-linearity. Such nonlinearity in certain approximations can be considered as the dissipative forces of interaction of excitation with the lattice. It will determine the condition $\dot{\mathbf{p}} \neq \mathbf{0}$ and the corresponding to this condition irregular dynamics of type of braking (or acceleration if exist a mechanism of energy selection by excitation from the environment of the crystal).

A condition $\dot{\mathbf{p}}=\mathbf{0}$ brings equation (17) over to the kind:

$$
\left(1 / 2 \mu_{\alpha}\right)\left(\partial^{2} \varphi_{f} / \partial \xi_{\alpha}^{2}\right)+g_{f} \varphi_{f}^{3}+\varepsilon_{f} \varphi_{f}=0,
$$

where it is marked:

$$
\varepsilon_{f} \equiv \Omega_{f}+\sum_{\alpha} 1 / \mu_{\alpha}, \quad \Omega_{f} \equiv \dot{\gamma}_{f}
$$

In this case $\varepsilon_{f}$ plays the role of the eigenvalue of the equation (18), and $\Omega_{f}$ has the meaning of the dimensionless frequency factor (corresponding to the dimensional notation usually is: $\omega_{f}$ ). It is the eigenvalue of the excited state of whole crystal. Furthermore, this condition $(\dot{\mathbf{p}}=\mathbf{0})$ leads to the fact that the momentum $\mathbf{p}$ is constant in time. Consequently, dynamic conditions (15), (16) it is possible to result to a concrete type: $\mathbf{r}_{0}=\boldsymbol{\beta} \tau$. Or in components: $x_{0}^{(\alpha)}=\beta_{\alpha} \tau=\tau \sin \left(p_{\alpha}\right)$.

With the permanent momentum $(\dot{\mathbf{p}}=\mathbf{0})$ equation (18) becomes fully stationary due to constancy of vector of speed: $\beta_{\alpha}=\sin \left(p_{\alpha}\right)$, and constancy of the component of tensor of mass:

$$
\mu_{\alpha} \equiv 1 / \cos \left(p_{\alpha}\right) \equiv 1 / \sqrt{1-\beta_{\alpha}^{2}} .
$$

Also it becomes automatically compatible with equation (11) and its solution in the form (14).

Analysis of solutions of the stationary equation (18) is the subject of many investigations (for example, $[1,3,4,13-18])$. Therefore here we will stop only on the main aspects of this problem and on one is relative a new solution. This new solution logically follows from the previous investigations and is useful for other types of nonlinearity.

As now the parameters of equation (18) are constants, this equation can be brought to the form:

$$
(1 / 2) \Delta \varphi_{f}(\boldsymbol{\rho})+g_{f} \varphi_{f}^{3}+\varepsilon_{f} \varphi_{f}=0
$$

by simple replacement of variables:

$$
\rho_{\alpha}=\xi_{\alpha} \sqrt{\mu_{\alpha}},
$$

where $\rho_{\alpha}$ are components of vector $\rho$, and $\Delta \equiv \sum_{\alpha} \partial^{2} / \partial \rho_{\alpha}^{2}$-the Laplas operator. Applying the methods of theory of differential equations, it is possible to show that it is impossible to build the analytical solutions of equation (21) in centrally symmetric case, when: $\Delta=\partial^{2} / \partial \rho^{2}+(2 / \rho)(\partial / \partial \rho)$, where:

$$
\rho=\sqrt{\sum_{\alpha} \rho_{\alpha}^{2}}
$$

Known only the numerical and asymptotic its solutions $[19,20]$.

But analytical solutions of this equation in one-dimensional case [18] are well known, where, for example: $\Delta \equiv \partial^{2} / \partial \rho_{z}^{2}$. Such solutions are called solitons and they have the form:

$$
\varphi_{f}\left(\rho_{z}\right)=B_{f} / \operatorname{ch}\left(\lambda_{f} \rho_{z}\right)
$$


Parameters $B_{f}, \lambda_{f}$ is determined by direct substitution of this solution in equation (21) with a taking into account the identity: $\Delta \equiv \partial^{2} / \partial \rho_{z}^{2}$. As a result of this substitution can be found: $\lambda_{f}^{2}=-2 \varepsilon_{f}$; $B_{f}^{2}=-2 \varepsilon_{f} / g_{f}$. Immediately visible that the eigenvalue $\varepsilon_{f}$ can only be negative: $\varepsilon_{f}=-\left|\varepsilon_{f}\right|$, and is determined by the normalization condition: $N_{f} \equiv \sum_{\mathbf{n}}\left|a_{f} \mathbf{n}\right|^{2}$. In the one-dimensional case it takes a particular form:

$$
\int_{-\infty}^{\infty} \varphi_{f}^{2}\left(\rho_{z}\right) d \rho_{z}=N_{f} \sqrt{\mu_{z}} .
$$

Usually considered $N_{f}=1$, and this condition gives: $\left|\varepsilon_{f}\right|=\left(g_{f}^{2} / 8\right) \mu_{z}$. For $B_{f}$ and $\lambda_{f}$ can be, respectively, obtained finally: $B_{f}=(1 / 2) \sqrt{g_{f} \mu_{z}} ; \lambda_{f}=\left(g_{f} / 2\right) \sqrt{\mu_{z}}$.

These solutions are so physically attractive that there is always a temptation to use it in the case of not one-dimensional spaces, which are defined by the general equation (21). In particular, this equation is satisfied by the following solutions: $\varphi_{f}(\boldsymbol{\rho})=B_{f} / \operatorname{ch}\left(\lambda_{f}^{x} \rho_{x}+\lambda_{f}^{y} \rho_{y}+\lambda_{f}^{z} \rho_{z}\right)$. These solutions do not have a spatial structure (are constants) in planes: $\lambda_{f}^{x} \rho_{x}+\lambda_{f}^{y} \rho_{y}+\lambda_{f}^{z} \rho_{z}=$ const, which are perpendicular to direction of distribution of soliton. On the other hand they have spatial configuration of soliton in the direction of distribution, which is perpendicular to the planes $\lambda_{f}^{x} \rho_{x}+\lambda_{f}^{y} \rho_{y}+\lambda_{f}^{z} \rho_{z}=$ const. Despite the fact that these solutions satisfy the equation (21), they however can not be acknowledged physical (at least in the quantum sense) because it does not have a finite norm.

To demonstrate this without loss of generality it is possible to do some simplification of the problem. Namely, we consider the motion only in $z$ direction. Then: $p_{x}=p_{y}=0, p_{z} \neq 0 ; \beta_{x}=\beta_{y}=0, \beta_{z}=\sin \left(p_{z}\right)$; $\mu_{x}=\mu_{y}=1, \mu_{z} \equiv 1 / \cos \left(p_{z}\right) \equiv 1 / \sqrt{1-\beta_{z}^{2}}$. Equation (18) then takes the form:

$$
(1 / 2)\left(\partial^{2} \varphi_{f} / \partial \xi_{x}^{2}\right)+(1 / 2)\left(\partial^{2} \varphi_{f} / \partial \xi_{y}^{2}\right)+\left(1 / 2 \mu_{z}\right)\left(\partial^{2} \varphi_{f} / \partial \xi_{z}^{2}\right)+g_{f} \varphi_{f}^{3}+\varepsilon_{f} \varphi_{f}=0,
$$

As can be seen, equation (26) is axially symmetrical relative to the axis $z$. If now to suppose that the unknown function $\varphi_{f}(\boldsymbol{\xi})$ does not depend on the components $\xi_{x}, \xi_{y}$ of the vector $\boldsymbol{\xi}$ (i.e., does not have the structure in a direction transverse to the directions of motion), the solution takes the form (24). A difference is only that now the solution depends on a variable $\xi_{z}$, instead of $\rho_{z}$. But the normalization condition (25) acquires another form now:

$$
\iiint_{(\infty)} \varphi_{f}^{2}\left(\xi_{z}\right) d \xi_{x} d \xi_{y} d \xi_{z}=N_{f} .
$$

It can be seen that left part of this equality is divergent. To ensure its convergence is necessary to limit integration along the directions $\xi_{x}, \xi_{y}$. Thus, such limitation must be executed, so that it did not conflict with equation (26) at least approximately. In general, it is impossible to represent the solution of nonlinear equations as multipliers. For example, to represent it in a form: $\varphi_{f}(\boldsymbol{\xi})=H_{f}\left(\xi_{\perp}\right) \Phi_{f}\left(\xi_{z}\right)$, where $\xi_{\perp}=\sqrt{\xi_{x}^{2}+\xi_{y}^{2}}$. But it turns out there is a physically consistent case when this can be done exactly in terms of generalized functions. This case corresponds to the special choice of multiplier $H_{f}\left(\xi_{\perp}\right)$ as the generalized function of Heaviside $H_{f}\left(\xi_{\perp}\right)=\theta_{-}\left(1-\lambda_{f}^{\perp} \xi_{\perp}\right)$. Or: $H_{f}\left(\xi_{\perp}\right)=1$ if $\lambda_{f}^{\perp} \xi_{\perp} \leq 1$ and $H_{f}\left(\xi_{\perp}\right)=0$ if $\lambda_{f}^{\perp} \xi_{\perp}>1$. A parameter $\lambda_{f}^{\perp}$ is the reverse radius of a region in which a function $H_{f}\left(\xi_{\perp}\right)$ is different from a zero, and outside of this region it equal to the zero. The problem of concrete value of parameter $\lambda_{f}^{\perp}$ was discussed, for example, in [13]. Such choice of multiplier $H_{f}\left(\xi_{\perp}\right)$ associated with two important properties, namely: $H_{f}^{3}\left(\xi_{\perp}\right)=H_{f}\left(\xi_{\perp}\right)$ and $\frac{\partial H_{f}}{\partial \xi_{\perp}}=0$. These properties give formal possibility to consider the representation $\varphi_{f}(\boldsymbol{\xi})=H_{f}\left(\xi_{\perp}\right) \Phi_{f}\left(\xi_{z}\right)$ as exact solution of equation (26) which has a finite norm. A multiplier $\Phi_{f}\left(\xi_{z}\right)$, in this case, is determined by equation: $\theta_{-}\left(1-\lambda_{f}^{\perp} \xi_{\perp}\right)\left\{\left(1 / 2 \mu_{z}\right) \Phi_{f}^{\prime \prime}\left(\xi_{z}\right)+g_{f} \Phi_{f}^{3}+\varepsilon_{f} \Phi_{f}\right\}=0$. This equation satisfied identically outside of region $\xi_{\perp}=1 / \lambda_{f}^{\perp}$ (i.e. at $\lambda_{f}^{\perp} \xi_{\perp}>1$ ) due to properties of $\theta$-function. Inside of this region (at $\lambda_{f}^{\perp} \xi_{\perp} \leq 1$ ) 
it is satisfied due to differential equation: $\left(1 / 2 \mu_{z}\right) \Phi_{f}^{\prime \prime}\left(\xi_{z}\right)+g_{f} \Phi_{f}^{3}+\varepsilon_{f} \Phi_{f}=0$, and creates the solutions in a form (24): $\Phi_{f}\left(\xi_{z}\right)=B_{f} / \operatorname{ch}\left(\lambda_{f} \xi_{z}\right)$. The full solution: $\varphi_{f}(\boldsymbol{\xi})=\theta_{-}\left(1-\lambda_{f}^{\perp} \xi_{\perp}\right) \Phi_{f}\left(\xi_{z}\right)$, with the taking into account the normalization conditions (27), but in a form: $\iiint_{f}^{2}(\boldsymbol{\xi}) d \xi_{x} d \xi_{y} d \xi_{z}=1$, becomes such:

$$
\varphi_{f}^{2}(\boldsymbol{\xi})=\mu_{z} \cdot\left(\left(\lambda_{f}^{\perp}\right)^{4} g_{f} / 4 \pi\right) \cdot \theta_{-}\left(1-\xi_{\perp} \lambda_{f}^{\perp}\right) \cdot c h^{-2}\left(\left(\left(\lambda_{f}^{\perp}\right)^{2} g_{f} / 2 \sqrt{\pi}\right) \mu_{z} \xi_{z}\right) .
$$

The square of solution is resulted here, because exactly it has the physical meaning and is called soliton.

Two circumstances attract the attention at once. First of all, product $\mu_{z} \xi_{z}$ in the argument of function $c h^{-2}(\ldots)$, at the account of explicit form of multipliers $\left(\mu_{z}=1 / \sqrt{1-\beta_{z}^{2}}, \xi_{z} \equiv z-z_{0}(\tau)\right.$, where $\left.z_{0}(\tau)=\beta_{z} \tau\right)$, acquires such explicit form: $\mu_{z} \xi_{z} \equiv\left(z-\beta_{z} \tau\right) / \sqrt{1-\beta_{z}^{2}}$. I.e., has a Lorentz-invariant form in dynamic direction. Secondly, all factor $\varphi_{f}^{2}(\boldsymbol{\xi})$ is proportional to the parameter $g_{f}$. In accordance with the definition (13), this parameter is inversely proportional to the matrix element $\left|M_{f}\right|$. In turn, the matrix element $\left|M_{f}\right|$, as defined in (10), is inversely proportional to the "rest mass"of a quasiparticle $m_{f}$. I.e., the function $\varphi_{f}^{2}(\boldsymbol{\xi})$ ultimately is proportional to mass $m_{f}$. This would be not so important, but function $\varphi_{f}^{2}(\xi)$, in accordance with (2), results in emergence of curvature in space of crystalline grate (around the point $z_{0}(\tau)=\beta_{z} \tau$ of localization of excitation). I.e., formally it looks so, as if mass of quasiparticle generates curvature of space of crystal. It is possible also to take into account, that a function $\varphi_{f}^{2}(\xi)$ is proportional to a component of dimensionless dynamic mass $\mu_{z}$. In this case a function $\varphi_{f}^{2}(\boldsymbol{\xi})$ will be proportional to the component of complete dynamical mass which is determined by the product $\mu_{z} m_{f}$.

Eigenvalue $\varepsilon_{f}$, appearing in equations (18), (21), (26) and similar, takes the form:

$$
\varepsilon_{f}=-\left(\mu_{z} g_{f}^{2}\left(\lambda_{f}^{\perp}\right)^{4} / 8 \pi\right) .
$$

If now introduce the notation: $\sigma_{f} \equiv\left(g_{f}^{2}\left(\lambda_{f}^{\perp}\right)^{4} / 8 \pi\right)$, the common eigenvalue, which is defined by the identities (19), can be represented in a more compact form:

$$
\Omega_{f}\left(p_{z}\right)=-2-1 / \mu_{z}-\sigma_{f} \mu_{z} \equiv-2-\cos \left(p_{z}\right)-\sigma_{f} / \cos \left(p_{z}\right) .
$$

By means of this relation it is possible already to determine dynamic properties [12] of quasiparticle. It is possible to show [13], that in approximation $\sigma_{f}<<1$ the complete wave function (5) with account (8) of the examined excitation will have the form of modulated by amplitude plane wave:

$$
\begin{gathered}
a_{f \mathbf{n}}(t) \Rightarrow a_{f}(\tau, \mathbf{r})=\varphi_{f}\left(\xi_{\perp}, \xi_{z}\right) \cdot \exp \left[i\left(p_{z} z-\Omega_{f}\left(p_{z}\right) \tau\right)\right] \equiv \\
\equiv \frac{\left(\lambda_{f}^{\perp}\right)^{2} \sqrt{g_{f}}}{2 \sqrt{\pi}} \cdot \frac{\theta_{-}\left(1-\xi_{\perp} \lambda_{f}^{\perp}\right)}{\sqrt[4]{1-\beta_{z}^{2}}} \cdot \frac{\exp \left(i p_{z}\left(z-\beta_{z} \tau\right)\right)}{c h\left(\sqrt{2 \sigma_{f}} \cdot \frac{z-\beta_{z} \tau}{\sqrt{1-\beta_{z}^{2}}}\right)} \cdot \exp (i S) \cdot \exp (i 2 \tau)
\end{gathered}
$$

Here, the variable $\xi_{z}$ is written in an explicit form: $\xi_{z}=z-\beta_{z} \tau$, and the action $S$ is defined as the product: $S=l \tau$, since free motion is examined. The Lagrangian $l$, with account of a factor $\sigma_{f}$, is determined by known [13] expression: $l\left(\beta_{z}\right)=\beta_{z} \arcsin \left(\beta_{z}\right)+\sqrt{1-\beta_{z}^{2}}$, and if to take into account a factor $\sigma_{f}$ becomes more complete: $l\left(\beta_{z}\right)=\beta_{z} \arcsin \left(\beta_{z}\right)\left(1-\frac{\sigma_{f}}{1-\beta_{z}^{2}}\right)+\sqrt{1-\beta_{z}^{2}}\left(1+\frac{\sigma_{f}}{1-\beta_{z}^{2}}\right)$. This Lagrangian describes the dynamics of the classical type with respect to the canonically conjugate pair of variables: with respect to the wave momentum $p_{z}$ and with respect to the localization point of excitation: $z_{0}(\tau)=\beta_{z} \tau$. The quantum part of the solution, which is determined by the multiplier:

$$
\exp \left(i p_{z}\left(z-\beta_{z} \tau\right)\right) / \operatorname{ch}\left(\sqrt{2 \sigma_{f}} \cdot \frac{z-\beta_{z} \tau}{\sqrt{1-\beta_{z}^{2}}}\right),
$$


formulated as seen from the last expression in own frame of reference with respect to point $z_{0}(\tau)=\beta_{z} \tau$. It appears, that the solution (28) has another interesting property. We are talking about the wave-particle duality, which is clearly present in the solution (28). Indeed, a wave function (28) is the plane wave modulated on amplitude. The nature of the modulation has a particle-like form which is called a soliton. More exactly square of this factor is called soliton. It is visible that this soliton moves uniformly in space without attenuation and independently of "behavior" of the phase, and also satisfy the Lorentz invariance. Depending on the relationship between the parameters $g_{f}$ and $\lambda_{f}^{\perp}$ this excitation can have a continuous set of spatial forms [13]: from oblate spheroid (along the direction of the dynamic $z$ ) up to elongate. Clearly, that it is possible to find the conditions under which excitation will have nearly spherical form.

But despite all of the attractive features of the proposed axial-symmetric solution, it has one disadvantage. This disadvantage is the absence of the actual solution depends on the variable $\xi_{\perp}=$ $\sqrt{\xi_{x}^{2}+\xi_{y}^{2}}$ in the direction that is transverse to the dynamic direction. This is due to the use of theta functions. Using theta functions as part of the wave function, by itself, does not raise any objections. But it can not provide an adequate description of the curvature of space of crystal in all directions.

That is why further will discussed a possibility of constructing centrally symmetric solutions in more general types of nonlinearity than cubic. Thus, the theta function is used only as a factor that provides only finite norm rather than the properties of the wave function.

\section{Solitonic excitations in solids. Total non-linearity of the fifth degree}

Now we consider the situation when series expansion of the function $G_{f}\left(\left|a_{f \mathbf{n}}\right|^{2}\right)$ takes into consideration following after $\left(\left|a_{f_{\mathbf{n}}}\right|^{2}\right)^{2} \equiv\left|a_{f_{\mathbf{n}}}\right|^{4}$ summand, if this function itself does not have any symmetry. Then there must be real a representation: $G_{f}\left(\left|a_{f \mathbf{n}}\right|^{2}\right)=-\left(G_{f}^{(1)} / 2\right)\left|a_{f \mathbf{n}}\right|^{4}-\left(G_{f}^{(2)} / 3\right)\left|a_{f \mathbf{n}}\right|^{6}$. The choice of signs in this representation provides physical correctness of function $G_{f}\left(\left|a_{f \mathbf{n}}\right|^{2}\right)$ for typical crystals and for the examined approximation. Doing further sequence of transformations similar to that which was performed between the formulas (3) - (21), we can obtain the equation:

$$
(1 / 2) \Delta \varphi_{f}(\boldsymbol{\rho})+g_{f}^{(2)} \varphi_{f}^{5}+g_{f}^{(1)} \varphi_{f}^{3}+\varepsilon_{f} \varphi_{f}=0 .
$$

Parameters $g_{f}^{(1)}, g_{f}^{(2)}$ are defined analogously to the parameter $g_{f}$, which, in turn, defined in (13). The components of the vector $\rho$, according to (22), defined by the sequence of equations:

$$
\rho_{\alpha} \equiv \xi_{\alpha} \sqrt{\mu_{\alpha}} \equiv\left(x_{\alpha}-\beta_{\alpha} \tau\right) \sqrt{\mu_{\alpha}} .
$$

In a centrally symmetric case, this equation becomes:

$$
(1 / 2)\left(\partial^{2} \varphi_{f} / \partial \rho^{2}\right)+(1 / \rho)\left(\partial \varphi_{f} / \partial \rho\right)+g_{f}^{(2)} \varphi_{f}^{5}+g_{f}^{(1)} \varphi_{f}^{3}+\varepsilon_{f} \varphi_{f}=0 .
$$

Accordant to $(23) \rho \equiv \sqrt{\sum_{\alpha} \rho_{\alpha}^{2}}$. Some aspects of this type of nonlinearity analyzed in [15]. There, in particular, shown that in spatially one-dimensional case the equation (29) also has solutions which are inversely proportional to the hyperbolic cosine. But at the same time one can also find solutions that are expressed in the terms of degree functions. Exactly these solutions will be considered here, but in more detail.

Thus, we seek the solution of equation (29) as follows:

$$
\varphi_{f}(\rho)=B_{f} / \sqrt{1+\lambda_{f} \rho^{2}} .
$$

Substituting (30) into (29), one can find the condition under which (29) is satisfied identically:

$$
g_{f}^{(1)}=0 ; \quad \varepsilon_{f}=0 ; \quad B_{f}^{4}=\left(3 \lambda_{f} / 2 g_{f}^{(2)}\right) .
$$


The first of these conditions states the fact that the solution (30) satisfies the equation (29) only when cubic nonlinearity is absent in the equation (29). According to the theory of differential equations, the requirement on the parameters of the equation means that the corresponding substitution is not the solution. But in this case it may also mean that the decomposition of function $G_{f}\left(\left|a_{f \mathbf{n}}\right|^{2}\right)$ into a power series on factors $\left|a_{f \mathbf{n}}\right|^{2}$ begins with a term: $-\left(G_{f}^{(2)} / 3\right)\left|a_{f \mathbf{n}}\right|^{6}$. In turn, this means that the function $G_{f}\left(\left|a_{f \mathbf{n}}\right|^{2}\right)$ must have odd symmetry. Further we will work within this assumption.

The second condition in $(31)\left(\varepsilon_{f}=0\right)$ means that the eigenvalue of the excited state of the crystal $\Omega_{f}$, according to $(19),(20)$, has the form: $\Omega_{f}=-\sum_{\alpha} 1 / \mu_{\alpha} \equiv-\sum_{\alpha} \cos \left(p_{\alpha}\right)$. It corresponds exactly to the eigenvalue for the free movement of the object of the classical type [12-14], i.e. quasiparticle.

Finally, the third condition in $(31): B_{f}^{4}=\left(3 \lambda_{f} / 2 g_{f}^{(2)}\right)$, binds unknown parameters $B_{f}$ and $\lambda_{f}$, leaving free only one of them. The solution (30) now becomes:

$$
\varphi_{f}(\rho)=\left(3 \lambda_{f} / 2 g_{f}^{(2)}\right)^{1 / 4} / \sqrt{1+\lambda_{f} \rho^{2}} .
$$

The presence of the free parameter $\lambda_{f}$ allows not only raise the question about the finiteness of the norm for this solution, but also on its equality to a unit. The use of this solution in the normalization condition (25), which takes the form: $\int_{0}^{\infty} \varphi_{f}^{2}(\rho) \rho^{2} d \rho=N_{f} \sqrt{\mu_{x} \mu_{y} \mu_{z}} / 4 \pi$, immediately demonstrates the problem. It consists in that the integral on the left side of the last equality is divergent at the upper limit. To get rid of this problem we may, as in Section 3, extend the solution by means of the theta multiplier. I.e., to build a solution in the form: $\varphi_{f}(\rho)=\theta_{-}\left(\rho_{n s}-\rho\right) \Phi_{f}(\rho)$. Substitution of this extension for $\varphi_{f}(\rho)$ in equation (29) leads to the fact that this equation is transformed to the form:

$$
\theta_{-}\left(\rho_{n s}-\rho\right)\left[(1 / 2)\left(\partial^{2} \Phi_{f}(\rho) / \partial \rho^{2}\right)+(1 / \rho)\left(\partial \Phi_{f}(\rho) / \partial \rho\right)+g_{f}^{(2)} \Phi_{f}^{5}+g_{f}^{(1)} \Phi_{f}^{3}+\varepsilon_{f} \Phi_{f}\right]=0
$$

In the area $\rho>\rho_{n s}$ the left side of this equality identically equal to zero due to the theta multiplier. In the area $\rho \leq \rho_{n s}$ the last equation reduces to (29) with respect to the function $\Phi_{f}(\rho)$. The parameter $\rho_{n s}$, in contrast to a similar parameter $1 / \lambda_{f}^{\perp}$ of section 3 , can be unambiguously determined. For example, it may be determined by the condition of exceeding of a signal (which is usually proportional to the function $\varphi_{f}^{2}(\rho)$ ) over a noise level $\delta$. In this consideration it can be a condition of exceeding the lattice constant changes above the level of the amplitude of its thermal or zero oscillation (this change, determined by the definitions (2), is the result of excitation). I.e., such condition reduces to: $\varphi_{f}^{2}(\rho) \geq \delta$, and the value of $\rho_{n s}$ is determined by the equation: $\varphi_{f}^{2}\left(\rho_{n s}\right)=\delta$. From determination (32) is obvious, that a condition: $\left(3 \lambda_{f} / 2 g_{f}^{(2)}\right)^{1 / 2} \gg \delta$, must be executed.

Thus, analyzed in this section the situation has the following features. First, find a spherically symmetric solution of the nonlinear Schrödinger equation with a general nonlinearity of the fifth degree (NSE $3+5)$. This solution has the asymptotic form: $\varphi_{f}(\rho) \Rightarrow 1 / \rho$, at $\rho \rightarrow \infty$, where $\rho$ is defined in $(23)$. Second, the dynamic properties of the considered excitation, which are determined by the eigenvalue $\Omega$, identical to the free relativistic particle [12]. Third, these solutions can make sense of the amplitude of the quantum mechanical wave function because it has a finite norm.

At the same time, the asymptotic behavior of $\varphi_{f}(\rho) \Rightarrow 1 / \rho$ is not completely satisfactory, since the physical meaning has the function $\varphi_{f}^{2}(\rho)$. In addition, it was shown that "the NSE $3+5$ "in the spherically symmetric case always comes down to "NSE $5+\ldots$ excluding all the symmetries of the function $G_{f}\left(\left|a_{f \mathbf{n}}\right|^{2}\right)$, except for the odd. This gives reason to consider more general non-linearity of the ninth degree (NSE $5+9$ ), which is the next after just considered (if we take into account the odd function $\left.G_{f}\left(\left|a_{f \mathbf{n}}\right|^{2}\right)\right)$. 


\section{Solitonic excitations in solids. Total non-linearity of the ninth degree}

Taking into account the oddness of function $G_{f}\left(\left|a_{f_{\mathbf{n}}}\right|^{2}\right)$, one can obtain a representation:

$$
G_{f}\left(\left|a_{f \mathbf{n}}\right|^{2}\right)=-\left(G_{f}^{(1)} / 2\right)\left|a_{f \mathbf{n}}\right|^{6}-\left(G_{f}^{(2)} / 3\right)\left|a_{f \mathbf{n}}\right|^{10}
$$

Signs in this expansion also selected due to the negativity of factor $G_{f}\left(\left|a_{f \mathbf{n}}\right|^{2}\right)$. Further, may be got an equation similar to equation (29): $(1 / 2)\left(\partial^{2} \varphi_{f} / \partial \rho^{2}\right)+(1 / \rho)\left(\partial \varphi_{f} / \partial \rho\right)+g_{f}^{(2)} \varphi_{f}^{9}+g_{f}^{(1)} \varphi_{f}^{5}+\varepsilon_{f} \varphi_{f}=0$. This equation is satisfied by the solution, similar to $(30): \varphi_{f}(\rho)=\theta_{-}\left(\rho_{n s}-\rho\right) B_{f} /\left(1+\lambda_{f} \rho^{2}\right)^{1 / 4}$. But at the same time here was taken into account the expansion by the theta multiplier. As a result in a region of $\rho \leq \rho_{n s}$ we will get the correlations similar to (31):

$$
\varepsilon_{f}=0 ; \quad B_{f}^{4}=\left(5 g_{f}^{(1)} / g_{f}^{(2)}\right) ; \quad \lambda_{f}=\left(40\left(g_{f}^{(1)}\right)^{2} / g_{f}^{(2)}\right)
$$

These correlations differ from (31). Now parameters $B_{f}$ and $\lambda_{f}$ are determinate. Therefore, in normalization condition, which takes the form: $N_{f}=\frac{4 \pi}{\sqrt{\mu_{x} \mu_{y} \mu_{z}}} \int_{0}^{\rho_{n s}} \phi_{f}^{2}(\rho) \rho^{2} d \rho$, can not be set $N_{f}=1$. The parameter $N_{f}$ now defined by this condition. The effective radius of the excitation $\rho_{n s}$ as in the previous section, defined by the condition $\varphi_{f}^{2}\left(\rho_{n s}\right)=\delta$. It has a deterministic value also.

The physical features of this solution are such. First, it also is spherically symmetric. But it has another, than in the previous case, asymptotic: $\varphi_{f}^{2}(\rho) \Rightarrow 1 / \rho$, at $\rho \rightarrow \infty$. This result deserves attention. In the applications a function $\varphi_{f}^{2}(\rho)$ usually determines the spatial distribution of the physical characteristics of the excited crystal. In particular, it determines potential of injected in the crystal of charge. As is known, all potentials interesting in physical sense have just the same asymptotic. In such interpretation of the function $\varphi_{f}^{2}(\rho)$ the radial component of the gradient operator: $\partial \varphi_{f}^{2} / \partial \rho$, determines the radial component of strength of the corresponding field. A detailed analysis shows that the derivative $\partial \varphi_{f}^{2} / \partial \rho$ is inversely proportional to the matrix element $\left|M_{f}\right|$. Consequently, according to (10), it is proportional to a mass $m_{f}$. In a section 3 , we will remind, mass was proportional to the square of wave function, instead of derivative. The dynamic properties, which are determined by the eigenvalue $\Omega_{f}$, are also identical to the free relativistic particle. The got solutions have also the quantum sense, as have a finite (but not equal 1) norm.

The complete solution similar (28) is determined by correlation:

$$
a_{f \mathbf{n}}(t) \Rightarrow a_{f}(\tau, \mathbf{r})=\varphi_{f}(\rho) \exp \left[i\left(p_{\alpha} x_{\alpha}-\Omega_{f} \tau\right)\right] \equiv B_{f} \frac{\theta_{-}\left(\rho_{n s}-\rho\right) \exp \left(i p_{\alpha} \rho_{\alpha} / \mu_{\alpha}\right)}{\left(1+\lambda_{f} \rho^{2}\right)^{1 / 4}} \exp (i S)
$$

The parameters $B_{f}$ and $\lambda_{f}$ are determined in (33), and the action $S$ is determined by equality: $S=l(\boldsymbol{\beta}) \tau$, in which: $l(\boldsymbol{\beta})=\sum_{\alpha}\left(\beta_{\alpha} \arcsin \left(\beta_{\alpha}\right)+\sqrt{1-\beta_{\alpha}^{2}}\right)$. The Lagrangian $l(\boldsymbol{\beta})$ can be obtained as in work [12]. Namely, at transition from "global" variables $x_{\alpha}$ to the "local" variables: $\rho_{\alpha}=\left(x_{\alpha}-\beta_{\alpha} \tau\right) \sqrt{\mu_{\alpha}}$, it is necessary to take into account next circumstances. First, to take into account common relationship between the Lagrangian and Hamiltonian, which is defined by equality: $l(\boldsymbol{\beta})=\beta_{\alpha} p_{\alpha}-h$ (p). Secondly, it is necessary to take into account that the relationship between velocity and momentum of a quasiparticle has a "standard"form: $p_{\alpha}=\beta_{\alpha} / \sqrt{1-\beta_{\alpha}^{2}}$. And thirdly, to use the fact that for quasiparticles performed "chain"of equalities:

$$
\Omega_{f}=-\sum_{\alpha} 1 / \mu_{\alpha}=-\sum_{\alpha} \cos \left(p_{\alpha}\right)=h(\mathbf{p})=l_{m}(\boldsymbol{\beta})=-\sum_{\alpha} \sqrt{1-\beta_{\alpha}^{2}}
$$




\section{Conclusions}

The excited states of materials with the structure of crystals were analyzed. However, unlike [12], the reaction of crystalline lattice on excitation was substantially taken into account. Since the account of the interaction of the excitation with the lattice leads to nonlinear Schrödinger equations, was considerec several possible nonlinearities, which differs from cubic. The possibility of constructing of analytica spherically-symmetric solutions with finite norm analyzed. The corresponding solutions were found. $\mathrm{Al}$ of these solutions were considered so that the quantum description of the excitation was formulated in the own frame of reference relative to the point of conditional localization of excitement. Dynamic properties of excitations (of quasiparticles) are practically identical to the dynamics of the free relativistic particle in detail analyzed in [12]. Obtained solutions demonstrate expressed corpuscular-wave duality. Indeed these solutions are the amplitude modulated plane wave. Herewith character of amplitude modulatior has a form similar to the particle. The square of this amplitude is called a soliton. This soliton uniformly moves in space regardless of "behavior" of phase part of solution and without attenuation. An important feature of these solutions is that their amplitudes (or squares of the amplitudes) have an asymptotic behavior of the type of $1 / \rho$, with $\rho \rightarrow \infty$.

\section{References}

1. A. M. Kosevich, Low Temp. Phys. 27, 513 (2001).

2. S. I. Pekar. Crystal Optics and Additional Light Waves, Naukova Dumka, Kiev (1982) [in Russian].

3. A.S.Davydov. Solitons in Molecular Systems, Boston, Kluwer Academic Publishers (1991).

4. Y.Natanzon, L.S.Brizhik, A.A.Eremko, Phys. Status Solidi (b), 244, 545 (2007).

5. E.G.Petrov, Ya.R.Zelinskyy, V.May, P.Hänggi, J. Chem. Phys, 127, 084709(2007).

6. Yu.B.Gaididei, V.M.Loktev, Ukr. Fiz. Zh., 50, 400 (2005).

7. Yu.B.Gaididei, V.M.Loktev, Phys. Status Solidi (b), 147, 307 (1998).

8. A.S.Davydov, Solid State Theory. Nauka, Moscow (1976) [in Russian].

9. K.P.Shukla, B.Eliasson, Usp. Fiz. Nauk, 180, 55 (2010).

10. K.P.Shukla, B.Eliasson, Rev. Mod. Phys, 81, 24 (2009).

11. I.M.Mryglod, V.M.Kuporov, Ukr. Fiz. Zh., 55, 1172 (2010).

12. A.D.Suprun, L.V.Shmeleva, Functional Materials, 19, 508 (2012).

13. A.D.Suprun. Dynamic Properties of the Single-electron Excitation of Nonlinear Crystals. PPC "Kier University. Kyiv (2008) [in Ukrainian].

14. A.D.Suprun, Bulletin of Kiev University. Physics, 27,18 (1986).

15. A.D.Suprun, Functional Materials, 8, 436(2001).

16. I.I.Lyashko, A.E.Grischenko, A.M.Fedorchenko, A.D.Suprun. Dopovidi Academy of Sciencesof the USSR, Ser A, $52(1985)$.

17. A.D.Suprun, Theor. Math. Phys. 57, 1141 (1983).

18. A.S.Davydov, Usp. Fiz. Nauk, 138, 603 (1982).

19. R.Y.Chiao, E.Garmire, C.H.Townes, Phys. Rev. Let., 13, 479 (1964).

20. A.C.Scott, Phys.Rev.A, 26, 578 (1982). 